\title{
Design and Analysis of a Magnetically Actuated and Compliant Capsule Endoscopic Robot
}

\author{
Sehyuk Yim and Metin Sitti*
}

\begin{abstract}
In this paper, we propose a compliant and tetherless magnetic capsule endoscopic robot. The proposed capsule robot has two key features. First, it has one extra degree of freedom axial contraction capability to perform additional tasks such as a drug releasing, a drug injection, or a biopsy. Also, design features of the magnetically deformed capsule robot are introduced. Its characteristic deformation curve, which was measured using an indentation setup, presents not only the deformation behavior of the magnetic capsule robot but also considerations in the capsule design process. Next, by implementing a magnetically actuated rolling locomotion scheme, the capsule can be controlled externally using a permanent magnet. The proposed magnetic capsule robot is anchored on a tissue wall by the magnetic attraction and rotated by a magnetic torque. This behavior allows a stable locomotion of the magnetic capsule robot and its orientation is controllable during locomotion. To verify the feasibility of proposed locomotion method and the compliant capsule's shape deformation, the magnetic capsule robot was actuated in a synthetic stomach model. The experimental results show that locomotion behavior of the capsule is stable and a successful tracking performance of the proposed magnetic actuation method; average distance gap between the capsule and the external magnet was only $20 \%$ of the capsule's body length. Such a soft and tetherless capsule robot can potentially enable minimally invasive diagnostic and treatment applications for stomach diseases.
\end{abstract}

\section{INTRODUCTION}

$\mathrm{C}$ URRENTLY, many researchers are working to develop the next generation capsule endoscope that actively locomotes [1]-[3] and performs biopsy [4], [5] and therapy types of new functions [6], [7]. This is motivated by the limited functions of the current passive capsule endoscopes [8]; because these capsule endoscopes cannot be actively controlled, these devices have no capabilities other than simple imaging.

Many groups suggested various capsule type robots as active capsule endoscopes. Approaches in these studies can be divided into two categories. First approach uses on-board actuation to self-propel capsule robots inside the digestive organs. For example, Park et al. proposed a robot using directionally deploying legs [1]. Next approach uses external actuation methods, such as electromagnetic coils or permanent magnets outside of the body, to propel capsule robots inside the body. Such magnetically actuated capsule

S. Yim is with the Department of Mechanical Engineering, Carnegie Mellon University, Pittsburgh, PA 15213 USA. (e-mail: sehyuky@andrew.cmu.edu).

M. Sitti is with the Department of Mechanical Engineering and Robotics Institute, Carnegie Mellon University, Pittsburgh, PA 15213, USA (e-mail: sitti@cmu.edu). robots can be called as magnetic capsule endoscopes (MCEs). Sendoh et al. proposed one rotary locomotion mechanism, and Carpi et al. used an external magnet to move a capsule robot with a magnetic shell [2], [3]. In industry, Siemens and Olympus, which were motivated by the feasibility of MCEs, launched a new capsule robot project using an external magnetic actuation method [9].

All of the existing capsule robots are rigid, which could cause issues of being trapped in junctions or could cause tissue damages if capsule robot exerts high forces to the tissues during locomotion or clamping. Moreover, current capsule robots mainly use swimming or tissue surface locomotion using spinning or sliding to navigate inside the digestive organs, which could have significant locomotion difficulties while operating in an inflated 3-D stomach surface. Therefore, this study proposes a new compliant capsule robot with a new rolling based surface locomotion principle to navigate inside an inflated 3-D stomach surface.

In Section II, the overall application scenario of this capsule robot inside an inflated stomach environment is described. In Section III, critical design features for compliance are introduced. In Section IV, the magnetic actuation scheme for rolling based locomotion is introduced and one potential application of magnetically actuated shape deformation is introduced. Finally, in Section V, the magnetic actuation platform is built and the proposed concepts, magnetically actuated locomotion, and shape deformation are evaluated experimentally on a synthetic stomach surface.

\section{APPLICATION SCENARIO}

Figure 1 shows the application scenario of the proposed compliant magnetic capsule robot inside a stomach surface. The doctor inflates the stomach using water first, and then the patient swallows the capsule robot. The doctor operates a large external permanent magnet to manipulate it using a robotic manipulator to manipulate it while looking at to camera images of the capsule in real-time. The capsule robot moves inside the stomach using the rolling locomotion. Because the capsule is always pulled toward to the tissue and anchored, it remains stable all the time. During endoscopy, the operator can intuitively estimate the position and orientation of the capsule because the external magnet and the capsule are symmetric. If an abnormal tissue is observed, the doctor stops the capsule immediately and performs biopsy and therapy using soft capsule's one extra degree of freedom axial contraction motion, which is actuated magnetically. After the endoscopy, the capsule is excreted by the passive peristalsis. 


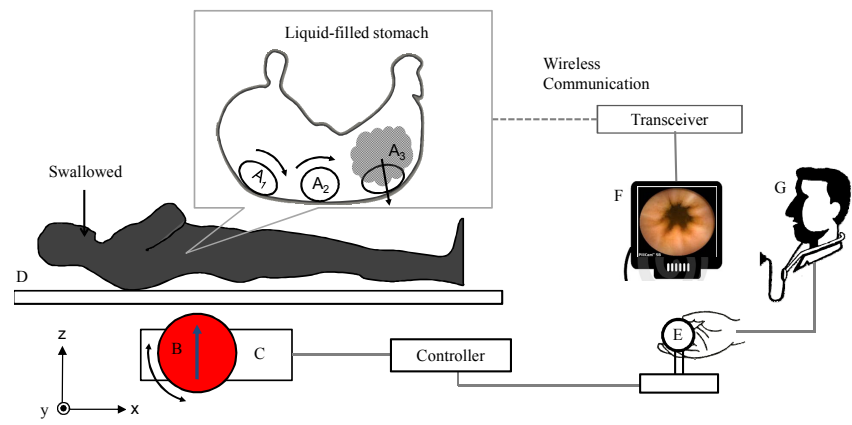

Fig. 1. Application scenario of the compliant magnetic capsule endoscopic robot: A: capsule endoscopic robot ( $\mathrm{A}_{1}-\mathrm{A}_{2}$ : rolling locomotion; $\mathrm{A}_{3}$ : biopsy or drug releasing; B: Spherical EPM (External Permanent Magnet); C: Motorized XYZ- $\theta$ stage; D: Bed for a patient; E: Joystick; F: Images and HMI (Human Machine Interface); G: Clinician.

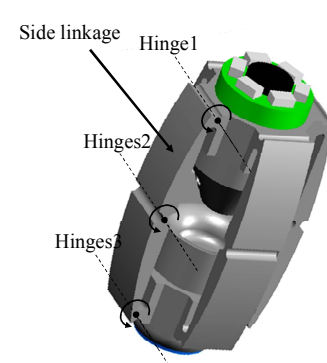

(a)

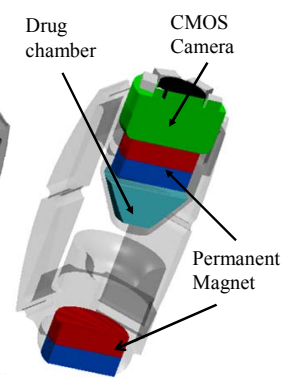

(b)
Fig. 2. The compliant capsule endoscopic robot: (a) Sarrus linkage mechanism; (b) Schematic of components

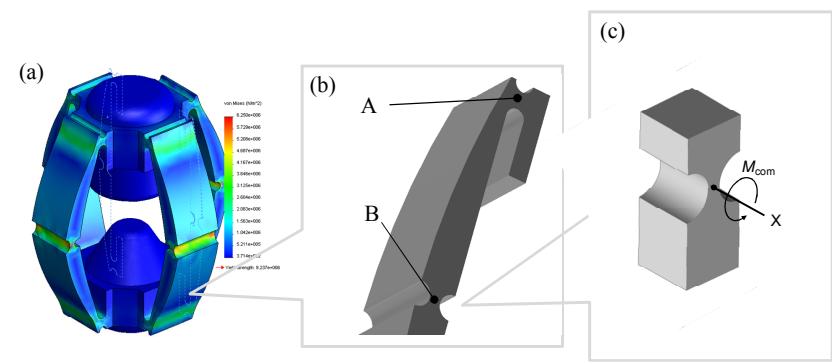

Fig. 3. Structure and geometry of compliant capsule robot: (a) Finite element analysis (COSMOS EXPRESS) on the capsule endoscope with the Sarrus linkages. The bright area represents the folded areas when the capsule is preloaded; (b) Geometry of the side linkage; A, C: circular flexure hinges, B:flexible beam, (c) Design variables of the circular flexure hinges: radius $r$, thickness $t$ and width $b$.

\section{COMPLIANCE DESIGN}

\section{A. Design Features}

Figure 2 shows the schematic of components on the compliant magnetic capsule robot. A camera module is implanted in the upper head. Two magnets are implanted in both ends to allow magnetic manipulation. A drug chamber is located below the magnet. The compliant magnetic capsule robot has three design features allowing magnetically actuated deformation and recovery process:

i) Sarrus linkage: The structure of the capsule is based on Sarrus linkage [10], [11]. With axial pre-load, the side linkages are folded and the capsule becomes fully compressed. If the preload force is removed, the capsule recovers its initial shape by the elastic restoring force of side linkages. ii) Circular flexure hinge: For the passive deformation and recovery of the capsule, the folded area by the magnetic attraction were designed as circular flexure hinges. As shown in Fig. 3(b), each side linkage has five flexible geometries; three circular flexure hinges and two flexible beams. A finite elements analysis of these parts is shown in Fig. 3(a). Among these, the circular flexure joint in the middle is the dominant factor determining the overall compliance because the compressive/tensile stresses are concentrated on this region. The actual values of these design variables in the compliant capsule are $700 \mathrm{~m}$ radius, $600 \mathrm{~m}$ thickness and $6 \mathrm{~mm}$ width.

iii) Polymer body: The whole body of the capsule is made of a soft elastomer, which allows it to be deformed by any environmental stimulus. The used elastomer material is a soft polyurethane (ST-1060, BJB Enterprise) with $2.07 \mathrm{MPa}$ Young's modulus. All the parts were manufactured by molding and attached to each other with the same polymer (ST-1060). Therefore, except for electronics and magnets, the whole body is monolithic. The fabrication process is as follows. First, master parts were made using a rapid prototyper (Invision HR, 3D System); the side linkage parts were manufactured using a micro-wire electrical discharge machine (AP 200L, Sodick) because of the complex geometry. Then, molds were made by pouring a silicone rubber (Dow Corning HS2, RTV rubber base for mold making) on the master parts. After the mold cured, the polyurethane was poured in the cavity of the mold. After the part cured, all parts were attached to each other using polyurethane. Finally, the camera module and magnets were inserted in the head.

\section{B. Compliance Characterization}

Due to the internal magnetic attraction, the capsule has a characteristic deformation behavior as shown in Fig. 4. According to the indentation experiment result, the resistive force of the capsule begins decreasing from a certain level of compressed length. Because the capsule is deformed making equilibrium between the magnetic attraction the resistive force, the deformation curve with large hysteresis loop is plotted. For example, at a preload force below $310 \mathrm{mN}$, the capsule is compressed continuously, but the stronger preload force makes the capsule fully compressed abruptly. In the relaxation, the capsule length is not increased even though the applied pre-load force is decreased. However, the capsule recovers its initial length almost if the pre-load falls below $160 \mathrm{mN}$. If the size of magnets decreases, the hysteresis weakens; the hysteresis loop area decreases, and the deformation curve with hysteresis converges to the deformation curve without the internal magnetic force, the blue curve.

Because the magnetic attraction is always present between head parts, ill-designed arrangement could result the permanent deformation induced by the internal magnetic attraction. Therefore, it is very important to maintain the condition for reversible deformation. Without an external magnet, if the magnetic attraction between internal magnets 


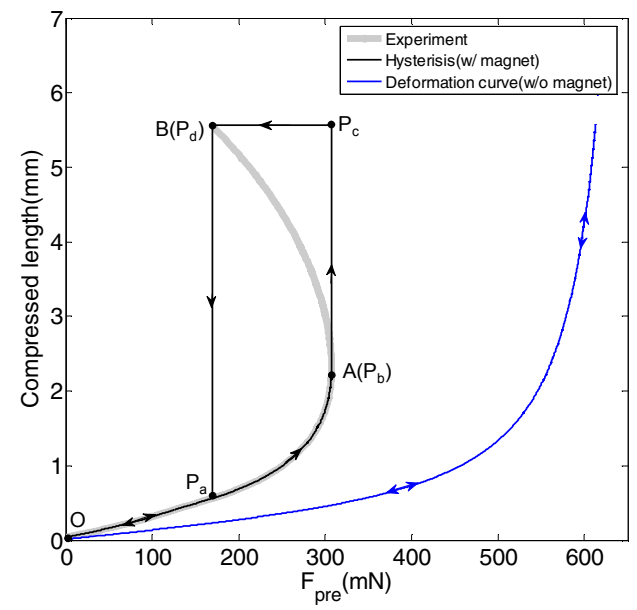

Fig. 4. Shape deformation curves of the compliant magnetic capsule and a dummy capsule (without internal magnets). Both capsules were compressed by an indenter and those resistive forces were measured using a load cell. The hysteresis curve was plotted based on the experimental data. Points A and $\mathrm{B}$ are critical points in the compression and relaxation motion. The cycle of the shape deformation (pre-loading and relaxation) is given with the order of O-Pa-Pb-Pc-Pd-Pa-O

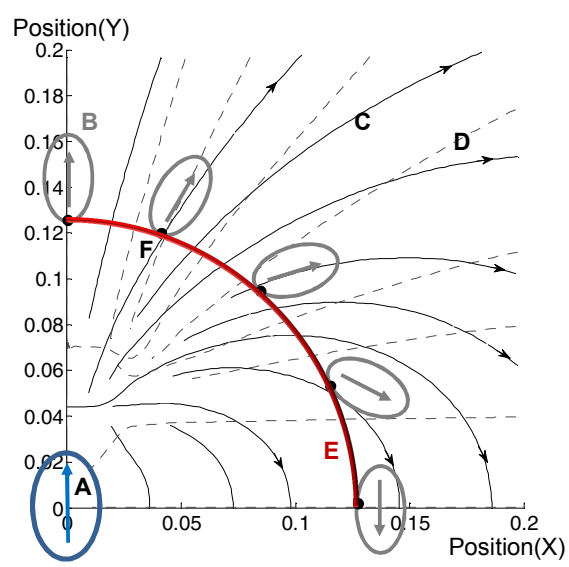

Fig. 5. Simulated magnetic field (flux density) and the magnetic attraction force vector. All the data was numerically calculated using MATLAB. A: The source magnet (Cylindrical magnet, diameter $50 \mathrm{~mm}$, length $80 \mathrm{~mm}$ ), B: The object magnet (Cylindrical magnet, diameter $5 \mathrm{~mm}$, length $5 \mathrm{~mm}$ ), C: The magnetic flux density line, D: The magnetic force line, E: The plane of symmetry (red band) F: Contact points.

overwhelms the restoring force at any time, then, the two head parts get stuck permanently. As the required conditions for the reversible shape deformation, first the magnetic attraction force between internal magnets should be lower than the resistive force of side linkages. Next, to implement the compressing motion of the capsule by the external magnetic field, the summation of the magnetic attraction between internal magnets and the external magnetic attraction on the upper head has to be larger than the resistive force. Thus, here are the conditions for the reversible capsule axial deformation:

$F_{m}^{i n}(h, B)<F_{\text {res }}^{s r s}(h) \leq F_{m}^{i n}\left(h, B_{\text {rein }}\right)+F_{m}^{e x}$

where the magnetic attraction force between internal magnets is $F_{\mathrm{m}}{ }^{\text {in }}(h, B)$, the capsule height $h$, the magnetic flux density passing through the center of magnets $B$, the resistive force of

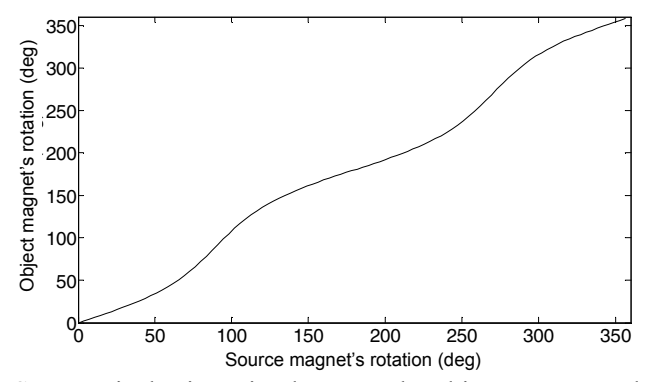

Fig. 6. Symmetrical orientation between the object magnet and the source magnet.

Sarrus linkages $F_{\text {res }}{ }^{\text {srs }}(h)$, the reinforced magnetic field by the external magnetic field $B_{\text {rein }}$ and the external magnetic attraction on the upper head $F_{\mathrm{m}}{ }^{\text {ex }}$.

The right condition of (1) can be satisfied if the external magnetic field is simply strong, but the left condition is related to the positions, size and magnetization of the internal magnets. To satisfy this condition, the force value at the critical point $\mathrm{B}$, the compressive force which is measured at the fully compressed capsule, should be positive. The negative compressive force represents that $F_{\mathrm{m}}{ }^{\text {in }}(h, B)$ is stronger than $F_{\text {res }}^{\text {srs }}(h)$. This is consistent with the analysis that too strong magnetic attraction induces a large hysteresis loop.

\section{Magnetic ACtuAtion}

\section{A. Symmetrical Orientation}

In micro-robotics, magnetic manipulation is a useful method not only for saving power source and space but also for controlling the capsule remotely [2], [3]. In this section, the magnetic actuation scheme, which was inspired by the symmetrical orientation between the capsule robot and the external magnet, is introduced in detail. Figure 5 shows the magnetic field and the magnetic force in 2-D, which are calculated using fundamental equations [12], [13]. It shows that the magnetic capsule's orientation about the plane of symmetry is symmetrical to the external magnet's one. The symmetry between those can be quantitatively verified by comparing rotational angles in Fig. 6. The capsule's rotational angle is approximately proportional to the external magnet's rotational angle, which means two magnets' orientations are symmetrical about the plane of symmetry.

\section{B. Rolling Locomotion Method}

The rolling locomotion actuated by magnetic torque is an original mechanism using the symmetrical orientation principle. Figure 7(a) shows the free body diagram of the magnetic capsule. It is anchored on the tissue surface by the vertical factor of the magnetic attraction $F^{m a g}$ and rotated by the magnetic torque, $T_{c}^{\text {mag }}$. Equation (2) and (3) show the trajectory of the center magnetic capsule robot governed by the magnetic force and the magnetic torque,

$$
F_{x}^{n e t}=F_{x}^{m a g}-\operatorname{sgn}\left(v_{c}-\frac{1}{2} h \dot{\theta}_{c}\right)\left[\mu\left(F_{z}^{m a g}+m g\right)\right]
$$




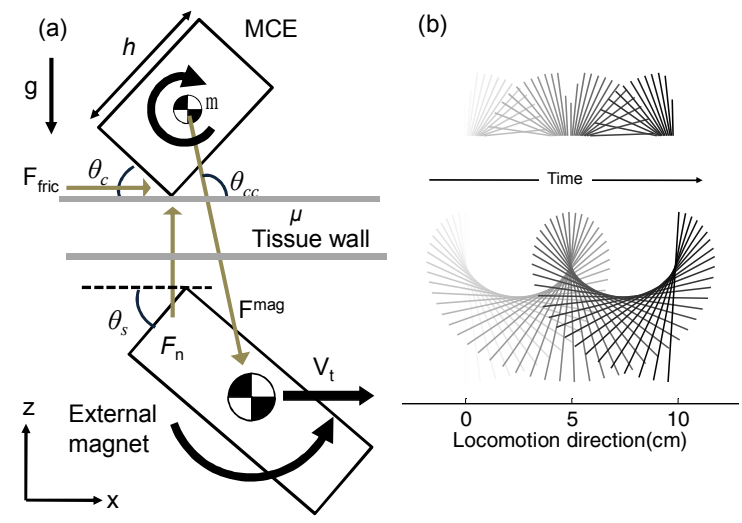

Fig. 7. (a) Free body diagram of the magnetically actuated rolling capsule robot; (b) Simulated rolling trajectory of the robot by the rotation of the external magnet. The external magnet and the capsule were simplified as lines. Traces are differently shaded according to time.

$$
\begin{aligned}
& \sum T_{c}^{n e t}=I_{c} \ddot{\theta}_{c}=T_{c}^{m a g}+\frac{1}{2} h\left(F_{z}^{m a g}+m g\right) \sin \theta_{c} \\
& -\left[\operatorname{sgn}\left(v_{P}^{r e l}\right) \mu\left(F_{z}^{m a g}+m g\right)\right] \cdot\left(\frac{1}{2} h \cos \theta_{c}\right)
\end{aligned}
$$

where $m$ is the mass of the capsule, $\mu$ the friction coefficient, $h$ the length of the capsule, $\underline{F}_{\mathrm{x}}^{\mathrm{mag}}\left(=F^{m a g} \cos \theta_{c c}\right)$ and $F_{\mathrm{z}}^{\mathrm{mag}}\left(=F^{m a g} \sin \theta_{c c}\right)$ the magnetic attraction in the $x$-axis direction and $z$-axis direction respectively, $\theta_{\mathrm{c}}$ the rotational angle of the capsule, $\theta_{\mathrm{s}}$ the rotational angle of the external magnet and $I_{\mathrm{c}}$ is the moment of inertia.

If the relative velocity between the contact area of the capsule and the tissue is zero, a static friction force is applied to the interface all the time. To align the capsule center with the external magnet center and make the locomotion stable using a static friction, the translational velocity of the external magnet should be set as,

$$
V_{t}(t)=r(t) \cdot \dot{\theta}_{c}(t) \cong r(t) \cdot \dot{\theta}_{s}(t)
$$

Figure 7(b) shows the simulated behavior of the capsule robot during one cycle. If the external magnet moves in the horizontal direction with rotated, the capsule is also navigated with being rotated by the magnetic torque.

This locomotion method has below advantages:

i) Continuous locomotion: The locomotion behavior of other magnetic capsule endoscopes, which are driven by a magnetic force while being dragged on a tissue surface, is similar to stick-slip motion because it starts moving when the horizontal magnetic attraction overcomes the static friction and stops when it falls below the dynamic friction. However, the rolling motion allows the capsule continuous locomotion because it moves using the static frictional torque at the contact area.

ii) Enhanced tracking performance: Other magnetic capsule endoscopes are dragged by the external magnet with a distance gap, because the horizontal magnetic force is maximized at the edge of external magnet. However, if the rolling capsule falls behind the external magnet, it uses both

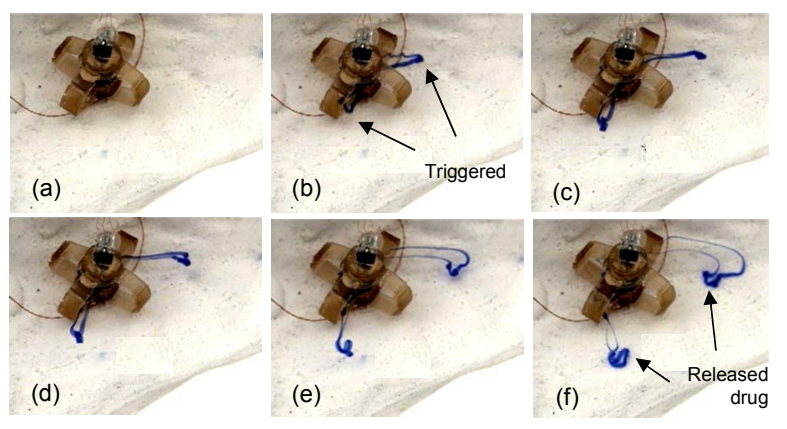

Fig. 8. Snapshots of the feasibility experiment using the second generation prototype (a)-(f): Motion sequence of drug releasing. Using the external magnet's controlled vertical motion, the robot creates a pressure that is higher than the threshold stress to open holes to release the liquid drug.
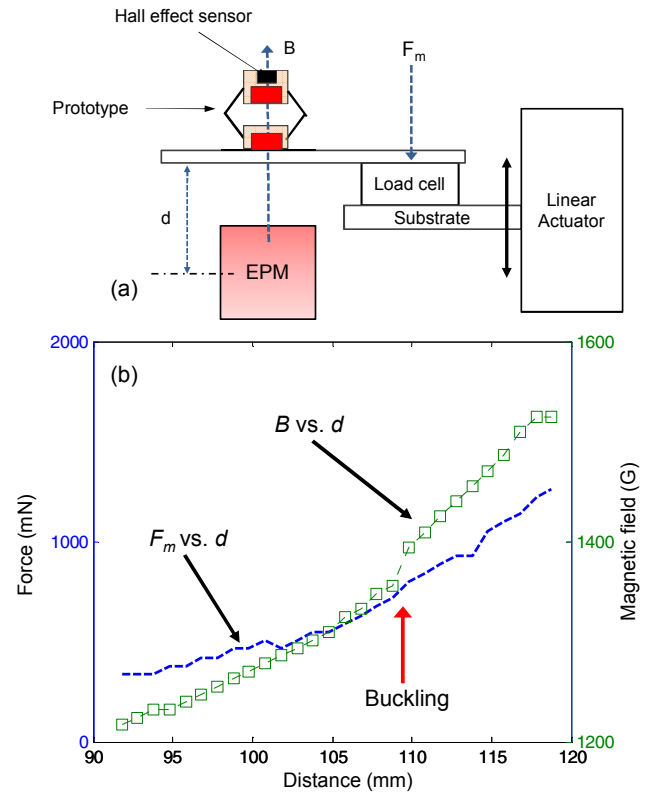

Fig. 9. (a) Experimental setup schematics to investigate: 1) the preload force between the capsule and the contact surface, Fm, as a function of the distance between the capsule and the external magnet, $d$; 2) the magnetic field B, measured by an on-board hall-effect sensor as a function the distance d.(b) Experimental force and magnetic field results for different distances.

the horizontal magnetic attraction and the friction force in the backward direction to track it.

iii) Posture control during locomotion: Other magnetic capsule endoscopes' posture can be controlled only in a static state. However, as shown in Fig. 7(b), the rolling magnetic capsule robot's one can be controlled using the symmetrical orientation principle even during locomotion. Some environmental disturbances such as a friction force or complex geometry of a tissue would temporarily cause an error between the desired orientation and the actual one. However, if the misalignment degree becomes larger, the magnetic alignment torque to decrease it is also reinforced. Therefore, the misalignment angle is automatically adjusted.

iv) Interface: Enhanced tracking performance and symmetrical orientation allows an intuitive interface between the capsule and the external magnet operator. The operator can estimate the position and orientation of the capsule watching the external magnet's ones. Endeavors for a complex positioning algorithm would be minimized. 


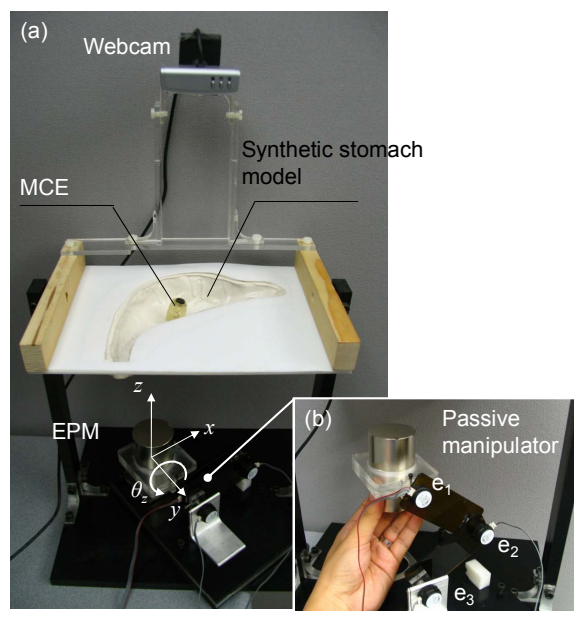

Fig. 10. Photographs of the experimental setup: (a) The synthetic stomach model, a rotational substrate, a web-camera, a source magnet, and a passive manipulator. (b) The passive manipulator with three encoders (S4, US digital) to calculate the position and orientation of the external magnet.
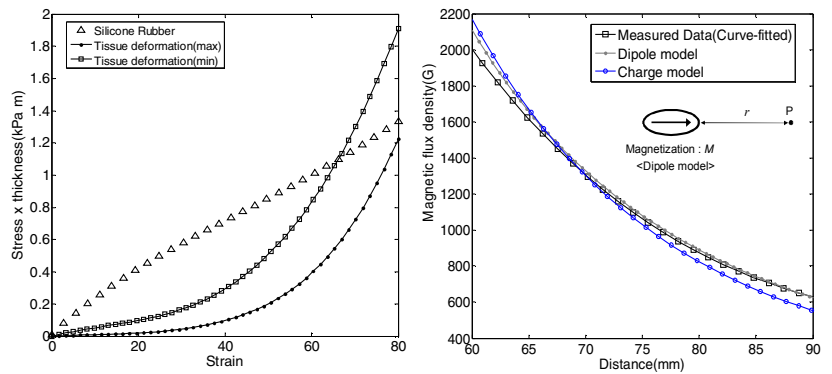

Fig. 11. (a) Comparison of the elongation property of a silicone rubber and a stomach tissue. (b) Magnetic flux density according to the distance from the external magnet. Data was measured using a hall-effect sensor (A1302, Allegro) and a linear actuator. The data over the maximum measurable value of the hall-effect sensor was estimated using a curve-fitting.

\section{Magnetically Actuated Shape Deformation}

One of the most important capabilities of the proposed capsule robot is its magnetically actuated axial compression motion. Figure 8 shows one use of this motion, drug releasing. Figures 8 show snapshots of the feasibility experiment using the second generation prototype. Here, the drug chamber is squeezed by the preload force of the convex area of the upper head due to the axial compression of the capsule by external magnetic forces. Then, the drug is released from the chamber through holes and layered on the target spot.

Figure 9 shows experiments to investigate the interaction force $F_{\mathrm{m}}$, the distance $d$, and the magnetic field B during the drug releasing. The preload force when the drug releasing was completed was about $850 \mathrm{mN}$. Also, $200 \mathrm{G}$ of external magnetic field was required for full squeezing of the chamber. The magnetic field used during attraction for the drug releasing can be completely decoupled from the magnetic field required for the capsule's locomotion. The detailed demonstration video of the drug releasing mechanism decoupled from the rolling locomotion can be seen in the multimedia file.

The advantage of active shape-morphing is to provide one extra degree of freedom motion in the vertical direction without any extra actuator. Considering that the robotic capsule endoscope for the next generation should perform biopsy and therapy, this extra degree of freedom motion can be used for these advanced functions.

\section{EXPERIMENTS}

\section{A. Characterization of the Experimental Setup}

Rolling locomotion experiments of the magnetically actuated compliant capsule robot were conducted to characterize its performance. Figure 10 shows the experimental setup. It consists of a synthetic silicone rubber stomach model, a rotational substrate, a data acquisition board, a web-cam, and a passive manipulator where the external magnet is mounted. A data acquisition board (USB $6008, \mathrm{NI})$ was used to record the data. The capsule position was measured in the captured images using a web-cam.

i) Stomach model: Even though it is difficult to simulate stomach's anisotropic and nonlinear properties, the degree of deformation can be simulated by adjusting the thickness of the stomach model. The elongation property of the silicone rubber specimen with $2.5 \mathrm{~mm}$ thickness and a human stomach tissue with $10 \mathrm{~mm}$ thickness, an average thickness of human stomach wall, are compared in Fig. 11(a); the original stress-strain data was multiplied with the thickness to include the effect of the thickness [14], [15]. In the range of from $0 \%$ to $45 \%$ strain, the deformation of the model is smaller than one of a real stomach tissue. From $46 \%$ to $68 \%$ strain, it is located between boundaries of tissue deformation. In a large strain area, over $80 \%$, the stomach model is less deformed than a real tissue.

ii) The magnetic property of the external magnet: Figure 11(b) shows the magnetic property of the external permanent magnet. There are two models to explain the magnetic property. One is the magnetic dipole model. According to this model, the magnetic flux density $B_{\text {axis }}$ in the magnetization direction at the point $P$ can be calculated as [16],

$B_{\text {axis }}(P)=\frac{\mu_{0}}{2 \pi r_{m}^{3}} V_{m} M$

where $r_{m}$ is the distance from the center of the magnet to the point $P, V_{\mathrm{m}}$ the volume of the magnet, $M$ is the magnetization, and $\mu_{0}$ is permeability. Based on this model, the magnetization of the external magnet was calculated as $1.45 \mathrm{MA} / \mathrm{m}$. Another one is the magnetic charge model. The residual induction $B_{\mathrm{r}}$ to match the model to the measured data was about 1.25T. As shown in Fig. 11(b), the dipole model was more accurate than the charge model.

\section{B. Rolling Locomotion Experiment}

The tracking performance of the capsule endoscope was evaluated. The capsule in the stomach simulator was actuated to move in $x$-axis direction. Figure 12 shows snapshots of the experiment. As expected in the simulation, the capsule was able to move in the stomach model using the rolling locomotion. Figure 13 shows the external magnet's position in the y-axis direction. Figure 13(a) shows the vertical position of the north pole and the south pole. The reason the length of the magnet seems to change in the figure is that the magnet is rotated about the $y$-axis. Around $5.2 \mathrm{sec}$ and 10.7 $\mathrm{sec}$, those become inverted. Considering the position of the 


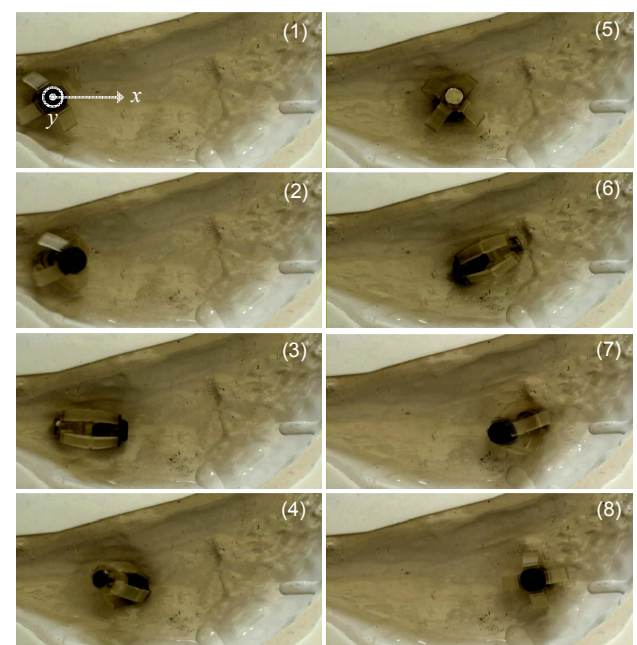

Fig. 12. Rolling locomotion experiment in the stomach simulator. Each number in images shows the sequence. The capsule endoscope moves in the $x$-axis direction with drawn in the y-axis direction. This axis reference is same as the reference of Fig. 10
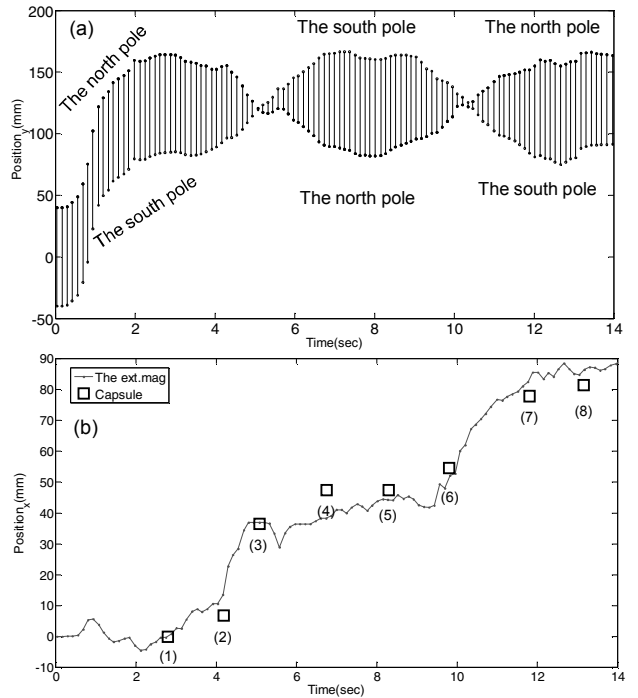

Fig. 13. Experimental positioning results: (a) Positions of the north pole and the south pole of the external magnet in the $z$-axis direction; (b) The center position of the external magnet in the $x$-axis direction, the locomotion distance. The position of the camera module in the capsule was measured using image analysis technique; the numbers below the capsule position data (square mark) corresponds to the numbers in Fig. 12.

substrate of the stomach model was $210 \mathrm{~mm}$, the effective range between the external magnet and the capsule, was about $90 \mathrm{~mm}$. Figure 13(b) shows the comparison of the locomotion distance of the external magnet and the capsule over time. It represents that the capsule can track the external magnet. At eight points in the experiment, the calculated center-to-center distance error was $5.88 \mathrm{~mm}$, which is $20 \%$ of the robot's body length.

\section{CONCLUSION AND FUTUREWORK}

In this paper, we proposed a new compliant capsule endoscope with one extra degree of freedom axial compression motion for medical procedures in stomach. Because this axial motion can be controlled by the external magnetic field, no extra actuator is needed. The proposed compliant capsule endoscope has a potential capability to monitor the whole area of the stomach in 3-D and to perform functions such as biopsy and drug delivery. Next, a magnetic actuation based rolling locomotion method for the capsule robot is proposed. By using this locomotion method, both the position and the orientation of the capsule can be controlled using an external permanent magnet. Future work of this research will focus on in vivo testing of the capsule robot and implementing various functions such as drug injection or biopsy.

\section{ACKNOWLEDGMENTS}

The authors would like to thank the other members of the CMU NanoRobotics Laboratory for their continuous feedback and suggestions, which have helped improving this work.

\section{REFERENCES}

[1] H. Park, S. Park, E. Yoon, B. Kim, J. Park, and S. Park, "Paddling based microrobot for capsule endoscopes," in Proc. IEEE Int. Conf. Robotics and Automation., pp. 3377-3382, 2007.

[2] M. Sendoh, K. Ishiyama, and K. Arai, "Fabrication of magnetic actuator for use in a capsule endoscope," IEEE Transactions on Magnetics, vol. 39, No. 5, pp.3232-3234, Sep. 2003

[3] Federico Carpi, Stefano Galbiati, and Angelo Carpi, "Controlled navigation of endoscopic capsule: concept and preliminary experimental investigations", IEEE Transactions on Biomedical Engineering, Vol. 54, No. 11, pp. 2028 - 2036, Nov 2007.

[4] Kong, K., Cha, J., Jeon, D., and Cho, D. "A rotational micro biopsy device for the capsule endoscope" in Proceedings of the IEEE/RSJ International Conference on Intelligent robots and systems, pp 1839 -1843 , Nov, 2005.

[5] S. Park et al, "A novel microactuator for microbiopsy in capsular endoscopes", Journal of Micromechanics and Microengineering, Vol. 18 , No.2, pp $250-260,2007$

[6] I. Wilding, P. Hirst, and A. Connor. "Development of a new engineering-based capsule for human drug absorption studies", Pharmaceutical Science and Technology Today, Vol. 3, pp.385-392, 2000.

[7] J. E. et al, "Implementation of multichannel sensors for remote biomedical measurements in a microsystems format" IEEE Transaction on. Biomedical. Eng., 2004, 51, Vol. 5, No. 3, pp. 525 535, 2004.

[8] Fireman $Z$ et al "Future of capsule endoscopy", Gastrointestinal Endoscopy Clinic, Vol. 14, No. 1, pp. 219-227, Jan, 2004.

[9] Available at http://www.siemens.com/press/

[10] Available at http://en.wikipedia.org/wiki/Sarrus_linkage

[11] A.M. Hoover and R.S. Fearing, "Analysis of off-axis performance of compliant mechanisms with applications to mobile millirobots", IEEE Int. Conf. Intelligent Robots and Systems, pp. 2770 - 2776, Oct. 2009.

[12] I. Choi , "Magnetic field Analysis and Experimental study on bowels characteristics for a capsule endoscope towed by Magnetic Force" M.S thesis, Korea aerospace university, December 2005.

[13] J. J. Abbott, O. Ergeneman, M. P. Kummer, A. M. Hirt, and B. J. Nelson "Modeling Magnetic Torque and Force for Controlled Manipulation of Soft-Magnetic Bodies", IEEE Transactions on Robotics, Vol. 23, No. 6, pp.1247-1252. December 2007.

[14] Yamada, M.D Strength of biological materials(Williams \& Wilkins, Baltimore), 1970.

[15] Sanghoon Park et al, "Stretching causes extensive changes of gastric submucosa: is it acceptable to define $500 \mu \mathrm{m}$ as the safe margin" 2008, Gut and Liver, Vol. 2, No. 3, 2008.

[16] D. K. Cheng, Field and Wave Electromagnetics, 2nd Edition. Addison-Wesley Publishing Company, Inc., 1992. 